\title{
Erratum to: Neighborhood matters: the impact of Hispanic ethnic density on future depressive symptoms 1-year following an ACS event among Hispanic patients
}

\author{
Ellen-ge D. Denton ${ }^{1} \cdot$ Jonathan A. Shaffer $^{2} \cdot$ Carmela Alcantara $^{3} \cdot$ Esteban Cadermil $^{4}$
}

Published online: October 30, 2015

(C) Springer Science+Business Media New York 2015

\section{Erratum to: J Behav Med DOI 10.1007/s10865-015-9679-5}

The values in Tables 1 and 2 were not aligned correctly in the original publication. It is now corrected with this Erratum.

The online version of the original article can be found under doi:10.1007/s10865-015-9679-5.

Ellen-ge D. Denton

ellenge.denton@csi.cuny.edu

1 Department of Psychology, City University of New York, College of Staten Island, 2800 Victory Blvd., Building 4S, Room 229, Staten Island, NY 10314, USA

2 University of Colorado Denver - Denver Campus, CLASPsychology, 3NC North Classroom, 1255 10th Street Mall, Denver, CO 80217, USA

3 Columbia University School of Social Work, 1255 Amsterdam Avenue, Room 810, MC 4600, New York, NY 10027, USA

4 Department of Psychology, Clark University, 950 Main Street, Worcester, MA 01610-1477, USA 
Table 1 Descriptives, individual- and neighborhood-level characteristics by gender and unadjusted associations of individual and neighborhood characteristics with 1-year depressive symptoms among 252 Hispanic participants with acute coronary syndrome

\begin{tabular}{|c|c|c|c|c|c|}
\hline $\begin{array}{l}\text { Individual-and-neighborhood } \\
\text { level characteristics }\end{array}$ & $\begin{array}{l}\text { Total sample } \\
\text { mean (SD) unless } \\
\text { otherwise noted }\end{array}$ & $\begin{array}{l}\text { Male mean } \\
(\mathrm{SD}) \text { or } \\
\mathrm{N}(\%)\end{array}$ & $\begin{array}{l}\text { Female } \\
\text { mean }(\mathrm{SD}) \\
\text { or } \mathrm{N}(\%)\end{array}$ & $\begin{array}{l}\text { Bivariate } \\
\text { correlation with } \\
\text { 1-year BDI score }\end{array}$ & $\begin{array}{l}\text { Bivariate } \\
\text { correlation } \\
\text { with } \Delta \text { BDI score }\end{array}$ \\
\hline \multicolumn{6}{|l|}{ Demographic factors } \\
\hline Age & $61.4(11.0)$ & $60.7(11.4)$ & $62.6(10.4)$ & $-0.14^{*}$ & $-0.14^{*}$ \\
\hline English fluency & $1.9(1.5)$ & $2.0(1.5)$ & $1.9(1.6)$ & 0.04 & -0.01 \\
\hline Education (years) & $10.4(4.4)$ & $10.8(4.6)$ & $9.8(3.9)^{*}$ & -0.22 & 0.03 \\
\hline Foreign-born & $261(80.1)$ & $162(62.1)$ & $99(37.9)$ & 0.05 & 0.05 \\
\hline Male sex, $N(\%)$ & $199(61 \%)$ & - & - & $0.20 * *$ & $0.20 * *$ \\
\hline \multicolumn{6}{|l|}{ Cardiovascular factors } \\
\hline Charlson comorbidity index & $1.7(1.7)$ & $1.4(1.5)$ & $2.1(1.9)^{* *}$ & $0.19 * *$ & -0.12 \\
\hline Left ventricular ejection fraction & $50.3(12.6)$ & $49.1(12.4)$ & $52.2(12.6)^{*}$ & 0.03 & $0.15^{*}$ \\
\hline GRACE prognostic risk score & $89.6(30.0)$ & $88.7(29.2)$ & $91.1(31.4)$ & -0.12 & $-0.24 * *$ \\
\hline Baseline BDI score & $9.2(8.2)$ & $6.7(5.9)$ & $12.8(10.0)^{* *}$ & $0.63 * *$ & $-0.45 * *$ \\
\hline 1-year BDI score & $7.1(8.2)$ & $5.5(6.2)$ & $9.5(10.0)^{* *}$ & - & $0.41 * *$ \\
\hline \multicolumn{6}{|l|}{ Neighborhood SES factors } \\
\hline Median income $(\$)$ & $41,556(24,483)$ & $41,251(21,411)$ & $42,029(28,686)$ & -0.09 & -0.03 \\
\hline $\begin{array}{l}\text { Households receiving public } \\
\text { assistance }(\%)\end{array}$ & $6.3(4.5)$ & $6.4(4.5)$ & $6.1(4.4)$ & 0.01 & 0.01 \\
\hline Hispanic density (\%) & $56.1(26.6)$ & $55.7(26.5)$ & $56.7(26.6)$ & 0.08 & -0.02 \\
\hline Residential density (N) & $2308.2(955.7)$ & $2318.4(942.6)$ & $2292.5(979.3)$ & -0.06 & -0.004 \\
\hline
\end{tabular}

GRACE global registry of acute coronary events, $B D I$ beck depression inventory

$* p<0.05 ; * * p<0.01$

Table 2 Descriptives for demographic, clinical, and neighborhood factors by tertile Hispanic ethnic density among 252 Hispanic participants with acute coronary syndrome

\begin{tabular}{|c|c|c|c|c|}
\hline $\begin{array}{l}\text { Demographic, clinical, and } \\
\text { neighborhood factors }\end{array}$ & $\begin{array}{l}\text { Low density } \\
\text { Mean (SD) or N (\%) }\end{array}$ & $\begin{array}{l}\text { Moderate density } \\
\text { Mean (SD) or N (\%) }\end{array}$ & $\begin{array}{l}\text { High density } \\
\text { Mean (SD) or N (\%) }\end{array}$ & $p$ \\
\hline \multicolumn{5}{|l|}{ Demographic factors } \\
\hline Age & $61.5(9.8)$ & $62.9(11.2)$ & $60.9(11.1)$ & 0.47 \\
\hline Male & $20(10.1)$ & $40(20.1)$ & $139(69.8)$ & 0.76 \\
\hline English fluency & $2.7(1.6)$ & $2.7(1.4)$ & $1.6(1.4)$ & $<0.001$ \\
\hline \multicolumn{5}{|l|}{ Education (years) } \\
\hline Foreign-born & $11.8(4.1)$ & $11.9(3.6)$ & $9.8(4.5)$ & $<0.001$ \\
\hline Cardiovascular factors & $18(6.9)$ & $49(18.8)$ & $194(74.3)$ & 0.002 \\
\hline Charlson comorbidity index & $2.1(1.7)$ & $1.7(1.8)$ & $1.6(1.7)$ & 0.33 \\
\hline Left ventricular ejection fraction & $53.0(12.2)$ & $51.4(12.9)$ & $49.6(12.5)$ & 0.30 \\
\hline GRACE prognostic risk score & $88.5(28.7)$ & $89.0(32.2)$ & $90.0(29.7)$ & 0.95 \\
\hline Baseline BDI score & $8.1(7.1)$ & $9.0(7.9)$ & $9.6(8.5)$ & 0.62 \\
\hline \multicolumn{5}{|l|}{ Neighborhood SES factors } \\
\hline Median income (\$) & $96,709(45,184)$ & $54,255(24,594)$ & $32,931(9560)$ & $<0.001$ \\
\hline Households receiving public assistance $(\%, \mathrm{SD})$ & $1.3(1.7)$ & $4.2(4.9)$ & $7.4(4.0)$ & $<0.001$ \\
\hline Hispanic density $(\%$, SD) & $5.2(3.0)$ & $24.0(8.9)$ & $70.3(14.4)$ & $<0.001$ \\
\hline Residential density (N, SD) & $1674.1(599.6)$ & $1729.6(787.6)$ & $2536.4(930.7)$ & $<0.001$ \\
\hline 1 year BDI score & $5.0(5.0)$ & $6.3(7.7)$ & $7.5(8.6)$ & 0.29 \\
\hline$\Delta$ BDI score & $-2.6(5.4)$ & $-2.3(5.5)$ & $-1.9(7.7)$ & 0.86 \\
\hline
\end{tabular}

$p$ values are based on the analysis of variance for continuous measures

$p$ values are based on the $\chi^{2}$ test for categorical measures

GRACE Global Registry of Acute Coronary Events, BDI beck depression inventory 